\title{
Microneedling for the Treatment of Scars: An Update for Clinicians
}

This article was published in the following Dove Press journal:

Clinical, Cosmetic and Investigational Dermatology

\section{Margit LW Juhasz' \\ Joel L Cohen ${ }^{1,2}$}

'University of California, Department of Dermatology, Irvine, CA, USA;

${ }^{2}$ AboutSkin Dermatology and

DermSurgery, Greenwood Village, CO, USA
Correspondence: Margit LW Juhasz University of California, Department of Dermatology, I 18 Med Surge I, Irvine, CA 92697, USA

$\mathrm{Tel}+1$ 718-309-7368

Emailmjuhasz@uci.edu
Background: Microneedling (MN) is used for the treatment of scars, amongst other indications. Although used in Asia and the Middle East for decades, related to the supposed lack of post-procedure pigmentary alterations even in darker skin types, MN only recently gained attention in the United States as an effective, well-tolerated aesthetic treatment.

Materials and Methods: A systematic review of the Medline database was completed using search terms "microneedle" or "microneedling" or "micro needle" or "micro needling" and "scar". Included articles were written in English and discussed the use of MN for the treatment of scars in human subjects.

Results: Fifty-eight studies were included for review, with a total of 1845 patients treated for acne scarring, hypertrophic or keloid scars, and those resulting from surgery, trauma, varicella or smallpox. $\mathrm{MN}$ and its counterpart fractional radiofrequency MN (FRF-MN) were used as monotherapy or in combination with topical, surgical or systemic modalities. $\mathrm{MN}$ and FRF-MN treatment resulted in clinical improvement of scar appearance from baseline. No serious adverse events occurred.

Conclusion: $\mathrm{MN}$ is a well-tolerated, minimally invasive procedure that can be used for the treatment of scars with a high level of patient satisfaction. Further clinical studies are needed to develop standardized treatment protocols.

Keywords: microneedling, laser, peel, platelet-rich plasma, scar

\section{Introduction}

Microneedling $(\mathrm{MN})$, or percutaneous collagen induction therapy, has been used within the dermatologic subspecialty for skin rejuvenation, skin tightening including treatment of striae, scar remodeling of the face and body, and hair growth. Due to the relative lack of post-inflammatory hyperpigmentation, $\mathrm{MN}$ is often considered an alternative to laser procedures in darker skin phototypes (Fitzpatrick IV through VI). Although MN has been a popular, minimally invasive, procedure performed in Asia and the Middle East, only recently has MN garnered attention in the United States (US). Since the first clinical descriptions of subcision and "needle dermabrasion" (using a tattoo gun without ink), the production of MN devices has flourished. ${ }^{1,2}$ In the US, $\mathrm{MN}$ devices exist as both rollers, stampers, and pens (electrically powered or otherwise), and can be combined with radiofrequency (RF) in an effort to deliver energy below the epidermal surface - known as fractional radiofrequency microneedling (FRF-MN), thus avoiding epidermal damage and subsequent dyspigmentation. ${ }^{3}$

$\mathrm{MN}$ devices vary based on their needle length (i.e. depth of skin penetration), diameter, density and material. Disposable needle tips are considered safer from an infectious risk standpoint, especially given the recent concern of bloodborne disease 
spread especially with the aptly named "vampire facials" for skin rejuvenation, ${ }^{3}$ and reusable home-use devices. Devices that allow for variation of needle length are advantageous in that varying penetration depths may be necessary to treat different areas of the face or body; sebaceous areas required deeper needle penetration compared to the forehead or periocular areas. Prior studies demonstrate a needle length of $1 \mathrm{~mm}$ as being the most desirable and accurate setting, whereas needle lengths of $3 \mathrm{~mm}$ may still only penetrate to a depth of 1.5 to $2.0 \mathrm{~mm}$.

Animal models and in vitro examination of human tissue demonstrate that $\mathrm{MN}$ creates micro-channels and micro-wounds at the level of the dermis breaking compact, thickened collagen and inducing the wound healing cascade. Micro-channels cause little epidermal damage making $\mathrm{MN}$ safe to use in darker skin phototypes. Gene expression profiling before and after $\mathrm{MN}$ treatment demonstrates an upregulation in type I collagen expression, as well as glycosaminoglycans, vascular endothelial growth factor (VEGF), fibroblast growth factor (FGF)-7, epidermal growth factor (EGF), and transforming growth factor (TGF)- $\beta$, all important signaling molecules for collagen production, as well as neovascularization. Tissue histology after $\mathrm{MN}$ shows thickened epidermis, and an increase in dermal collagen and elastic fiber deposition. Over a period of weeks to months, newly formed type III collagen becomes mature type I collagen causing skin tightening and a decrease in the appearance of scars or rhytides. ${ }^{6-11}$ In this systematic review, we will explore the efficacy and safety of MN for the treatment of scarring.

\section{Materials and Methods}

A systematic review using the PRISMA (Preferred Reporting Items for Systemic Review and MetaAnalysis) was completed using the Medline database in August 2020 using the search terms "microneedle" or "microneedling" or "micro needle" or "micro needling" and "scar". Included manuscripts were case reports, case series and trials discussing the use of microneedling (rolling device, stamping device, fractional radiofrequency device) for the treatment of scars in humans in English. Reviews, animal models, and in vitro studies were excluded, as were articles not available in English.

\section{Results}

Search terms revealed a total of 246 manuscripts; after removal of duplicates, 58 studies ranging in date from 2011 to August 2020 were included for review. A total of 1845 patients with acne scarring or acne vulgaris, hypertrophic or keloid scarring, post-surgical or posttraumatic scars, and varicella or smallpox scarring completed study treatment protocol and follow-up. Protocols utilized included MN or FRF-MN alone, as well as in combination with topicals, chemical peels, subcision, platelet-rich plasma (PRP), laser, injectables or systemic medications. MN devices were both automated or mechanical in nature (such as rollers) (Table 1). ${ }^{6-63}$

In all cases of MN or FRF-MN treatment, patients received pre-treatment anesthesia. Although the most commonly reported anesthesia was topical (lidocaine and/or prilocaine) applied in the area to be treated with or without occlusion for a total of 30 to 90 minutes, 2 studies originating from Germany mentioned the use of general anesthesia. Prior to starting $\mathrm{MN}$, areas were cleansed with either isopropyl or ethyl alcohol. Two studies utilizing FRF-MN used epidermal cooling to decrease epidermal damage during the procedure. Post-treatment regimens suggested included sunscreen ( $\mathrm{n}=19$ studies), bland emollient $(\mathrm{n}=11)$, topical or systemic antibiotic including topical fusidic acid ( $\mathrm{n}=11)$, topical corticosteroids $(n=3)$, cold packs $(n=2)$, as well as topical benzoyl peroxide, cyclopentasiloxane/cyclohexasiloxine/sodium hyaluronate, hyaluronic gel, L ascorbic acid/ $\alpha$-tocopherol /ferulic acid, non-steroid anti-inflammatory drugs (NSAIDs), and tretinoin/kojic acid/hydroquinone/hydrocortisone ( $\mathrm{n}=1$ each). ${ }^{6-63}$

Acne scarring was by far the most discussed condition ( $n=43$ studies). In all studies, boxcar (U-shaped) and rolling (M-shaped) scars demonstrated the greatest clinical improvement after MN or FRF-MN, while icepick (V-shaped) scars were often recalcitrant. ${ }^{6,9,11-51}$ Patients treated with MN or FRF-MN for other types of scars also demonstrated clinical improvement. Scar improvement was measured using both patient and investigator qualitative assessments, as well as the Echelle d'evaluation Clinique des Cicatrices d'Acne (ECCA), Vancouver Scar Scale (VSS), and Visual Analog Scales (VAS). ${ }^{6-63}$ Combination treatment with laser, PRP, subcision, glycolic acid peel, Jessner's peel, trichloroacetic acid peel and topical amniotic fluid stem cells resulted in greater scar improvement than $\mathrm{MN}$ or FRF-MN alone. $^{8,17,24,26,31,32,34,37,40,51,53,55}$ Fifty to $100 \%$ of patients were satisfied with MN or FRF-MN treatment; $33 \%$ of patients reported they would want further treatment, ${ }^{9}$ while $94 \%$ would recommend treatment to others. ${ }^{28}$ Combining treatment with $1550 \mathrm{~nm}$ laser or PRP resulted 
Table I Summary of Protocols from Studies Discussing the Use of Microneedling for Scar Treatment

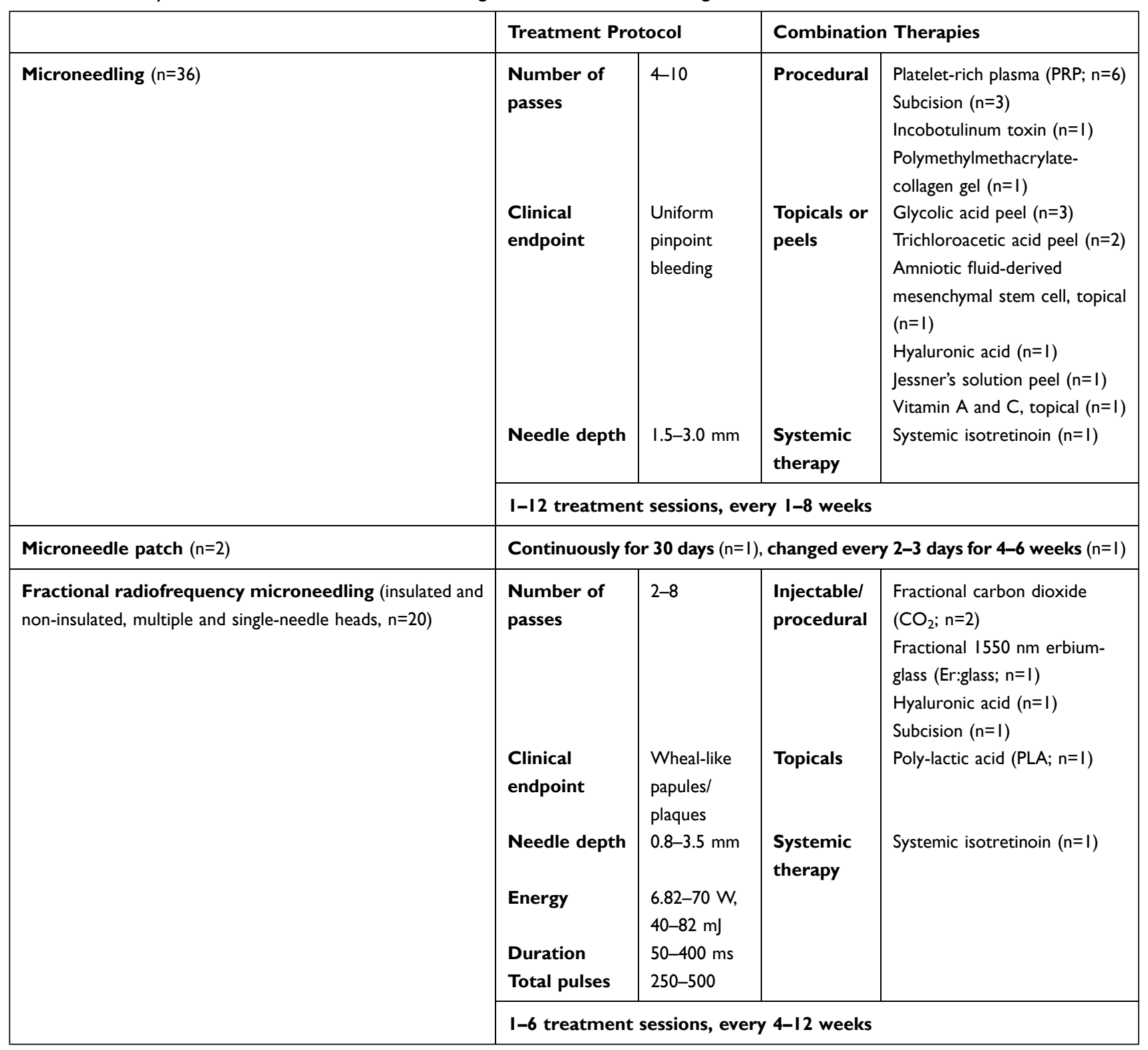

in higher patient satisfaction. ${ }^{8,34}$ In addition, patients preferred MN to intralesional triamcinolone (ILTAC) or 1450 nm diode laser. ${ }^{30,56}$

Adverse events (AEs) due to MN or FRF-MN were mostly of minimal severity; no serious AEs were reported. Almost all studies reported pain and bleeding during the procedure; the most common post-procedure AEs included transient post-procedure pain/discomfort/burning, erythema and/or swelling. Further AEs are discussed in Table 2. Post-inflammatory hyperpigmentation occurred in 19 studies; $54.5 \%$ of studies used FRMN, while $45.5 \%$ used MN. There was one case of herpes simplex reactivation which was successfully treated with oral valacyclovir.
The most feared $\mathrm{AE}$ of $\mathrm{MN}$ treatment is the so-called "railroad" or "tramtrack" scarring that can occur with aggressive treatment, and was only reported as an AE in 5 studies. $^{6-63}$ One female patient with atrophic acne scars developed scarring after an allergic reaction to the nickel contained in the needles, which was subsequently treated with oral prednisolone and topical steroids. ${ }^{38}$

\section{Discussion}

MN has gained popularity as a minimally invasive aesthetic technique for the treatment of skin aging, scarring, striae, and hair loss, amongst other indications. Although the US Food and Drug Administration (FDA) initially 
Table 2 Summary of Rare AEs Occurring with Microneedling Treatment for Scars

\begin{tabular}{|l|l|}
\hline Adverse Event Description & $\begin{array}{l}\text { Number of Studies Reporting } \\
\text { (n) }\end{array}$ \\
\hline Post-inflammatory & 19 \\
hyperpigmentation & \\
Scabbing/crusting & 9 \\
Purpura/ecchymosis & 5 \\
"Tramtrack"/“railroad" scarring & 5 \\
Acne flares & 4 \\
Cervical lymphadenopathy & 3 \\
Milia & 2 \\
Pustules/bullae & 2 \\
Allergic reaction to nickel & 1 \\
Herpes simplex reactivation & 1 \\
\hline
\end{tabular}

classified MN as class I medical devices, recent developments have elevated their classification to class II (special controls) and they are currently approved for microdermabrasion, scarring and rhytides. ${ }^{64}$ The literature suggests that MN and FRF-MN are well tolerated and result in clinical improvement of scarring due to acne or other infectious cause, hypertrophic or keloid scars, and postoperative or traumatic scars, as well as high rates of patient satisfaction. MN and FRF-MN were reportedly tolerated better by patients than their laser resurfacing counterparts, namely the $\mathrm{CO}_{2}$, Er:glass and diode lasers, with less reported downtime. MN and FRF-MN can be combined with a variety of other surgical therapies including laser resurfacing, chemical peels, PRP, filler and botulinum toxin for greater clinical results. ${ }^{6-63}$

As with many aesthetic procedures, MN and FRF-MN suffer from a lack of standardized protocol. Animal and human studies suggest that multiple passes per treatment and multiple treatment sessions demonstrate greater skin regeneration potential. ${ }^{65,66}$ Further clinical studies need to be completed to determine optimal number of passes, number of treatment sessions, intertreatment duration intervals and maintenance therapy.

Just like non-ablative laser techniques, $\mathrm{MN}$ is an effective intraepidermal and intradermal delivery method for pharmaceuticals. ${ }^{5,67}$ In addition to microneedles designed to contain substances such as bleomycin or triamcinolone for treatment hypertrophic scarring, ${ }^{68,69} \mathrm{MN}$ can enhance the penetration of topicals such as anesthetics, chemical peels, PRP or filler material such as hyaluronic acid as evidenced by this review, ${ }^{6-63}$ and possibly nanoparticles and siRNA in the future. ${ }^{67,68}$ Advances in MN delivery, such as the development of patches or use of MN to deliver energy sources below the level of the epidermis have both decreased the amount of discomfort associated with treatment, and increased efficacy by combining multiple treatment modalities. ${ }^{56,58,70}$ It is important to note that certain topical products may cause allergy, and even granulomatous reaction, when introduced into the skin through micro-channels created by MN devices; physicians should counsel patients appropriately regarding these risks. Substances such as bleomycin, triamcinolone and filler material should presumably be safer to administer through micro-channels given that they are designed as injectables.

Although no serious AEs were associated with $\mathrm{MN}$ and FRF-MN treatment of scars, it is important to note that post-inflammatory hyperpigmentation occurred in over $30 \%$ of studies and resolved either spontaneously or with the help of topical bleaching creams within months. Given that the majority of patients treated in this review had a Fitzpatrick skin phototype of IV or greater, AE reporting may have been biased towards events that more commonly occur in this patient groups post-procedure, specifically dyspigmentation and aberrant scarring (such as the "tramtracks"). Physicians should be aware that MN is not without its risks, and appropriately counsel patients during the consent process to avoid patient morbidity post-treatment.

Limitations of this study include its lack of metaanalysis. Given the heterogeneity of data presented by included studies, it was not possible to combine and statistically analyze this data.

\section{Conclusions}

MN and its relative FRF-MN are both well-tolerated, minimally invasive procedures that can be used for the effective treatment of scarring. Although there are no standard treatment protocols, clinical improvement in many types of scars including acne, varicella and smallpox, hypertrophic or keloid, and post-operative or posttraumatic scars have been reported in the literature. MN and FRF-MN can be used as stand-alone modalities, or can be combined with a variety of topicals and other surgical procedures for superior results. No serious AEs have been reported using MN or FRF-MN for the treatment of scars; however, physicians should be aware that postinflammatory hyperpigmentation is still a relatively common event. Large-scale, clinical trials need to be completed to determine optimal, standardized protocols to treat scarring using MN or FRF-MN. 


\section{Funding}

The authors received no funding for this research.

\section{Disclosure}

Dr. Cohen has been a consultant and participated in clinical research for Lutronic (the manufacturer of Genius fractional bipolar radiofrequency system). The auhtors report no other conflicts of interest in this work.

\section{References}

1. Camirand A, Doucet J. Needle dermabrasion. Aesthetic Plast Surg. 1997;21:48-51. doi:10.1007/s002669900081

2. Orentreich DS, Orentreich N. Subcutaneous incisionless (subcision) surgery for the correction of depressed scars and wrinkles. Dermato Surg. 1995;21(6):543-549. doi:10.1111/j.1524-4725.1995.tb00259.x

3. Howard J 'Vampire facial' at New Mexico spa tied to 2 HIV cases, health officials say. 2019. Available from: https:/www.cnn.com/ 2019/04/30/health/vampire-facial-hiv-cases-new-mexico-bn/index. html. Accessed December 4, 2020.

4. Lima E, Lima M, Takano D. Microneedling: experimental study and classification of the resulting injury. Surg Cosmet Dermatol. 2013;5:110-114.

5. Sasaki GH. Micro-needling depth penetration, presence of pigment particles, and fluorescein-stained platelets: clinical usage for aesthetic concerns. Aesthet Surg J. 2017;37:71-83. doi:10.1093/asj/sjw120

6. Moftah NH, El Khayyat MAM, Ragai MH, Alaa H. Carboxytherapy versus skin microneedling in treatment of atrophic postacne scars: a comparative clinical, histopathological, and histometrical study. Dermatol Surg. 2018;44(10):1332-1341. doi:10.1097/DSS.0000000 000001560

7. Busch K-H, Aliu A, Walezko N, Aust M. Medical needling: effect on skin erythema of hypertrophic burn scars. Cureus. 2018;10:e3260.

8. Ibrahim ZA, El-Ashmawy AA, Shora OA. Therapeutic effect of microneedling and autologous platelet-rich plasma in the treatment of atrophic scars: a randomized study. J Cosmet Dermatol. 2017;16 (3):388-399. doi:10.1111/jocd.12356

9. Osman MAR, Shokeir HA, Fawzy MM. Fractional erbium-doped yttrium aluminum garnet laser versus microneedling in treatment of atrophic acne scars: a randomized split-face clinical study. Dermatol Surg. 2017;16(Suppl 3):S47-S56. doi:10.1097/DSS.000000000000 0951

10. Min S, Park SY, Yoon JY, Suh DH. Comparison of fractional microneedling radiofrequency and bipolar radiofrequency on acne and acne scar and investigation of mechanism: comparative randomized controlled clinical trial. Arch Dermatol Res. 2015;307:897-904. doi:10.1007/s00403-015-1601-z

11. El-Domyati M, Barakat M, Awad S, Medhat W, El-Fakahany H, Farag H. Microneedling therapy for atrophic acne scars: an objective evaluation. J Clin Aesthet Dermatol. 2015;8:36-42.

12. An MK, Hong EH, Suh SB, Park EJ, Kim KH. Combination therapy of microneedle fractional radiofrequency and topical poly-lactic acid for acne scars: a randomized controlled split-face study. Dermatol Surg. 2020;46:796-802. doi:10.1097/DSS.0000000000002175

13. Kim J, Lee YI, Kim J, et al. Safety of combined fractional microneedle radiofrequency and $\mathrm{CO}_{2}$ as an early intervention for inflammatory acne and scarring treated with concomitant isotretinoin. Dermatol Surg. 2020;Publish Ahead of Print. doi:10.1097/DSS.000 0000000002364

14. Vijaya Lakshmi Y, Swetha Reddy L, Naga Neelima Devi K, et al. Evaluation of microneedling therapy in management of facial scars. J Craniofac Surg. 2020;31(2):e214-e217. doi:10.1097/SCS.00000 00000006145
15. Alster TS, Li MKY. Microneedling of scars: a large prospective study with long-term follow-up. Plast Reconstr Surg. 2020;145 (2):358-364. doi:10.1097/PRS.0000000000006462

16. Tatlıparmak A, Aksoy B, Shishehgarkhaneh LR, Gökdemir G, Koç E. Use of combined fractional carbon dioxide laser and fractional microneedle radiofrequency for the treatment of acne scars: a retrospective analysis of 1-month treatment outcome on scar severity and patient satisfaction. J Cosmet Dermatol. 2020;19(1):115-121. doi:10.1111/ jocd.13004

17. Biesman BS, Cohen JL, DiBernardo BE, et al. Treatment of atrophic facial acne scars with microneedling followed by polymethylmethacrylate-collagen gel dermal filler. Dermatol Surg. 2019;45(12):1570-1579. doi:10.1097/DSS.0000000000001872

18. Darmawan H, Kurniawati Y. Split-face comparative study of microneedling with platelet-rich plasma versus microneedling alone in treating acne scars. Skinmed. 2019;17:207-209.

19. Bhargava S, Kumar U, Varma K. Subcision and microneedling as an inexpensive and safe combination to treat atrophic acne scars in dark skin: a prospective study of 45 patients at a tertiary care center. J Clin Aesthet Dermatol. 2019;12:18-22.

20. Khalid FA, Ahmad S, Mehrose MY, et al. Efficacy of micro-needling on post acne scars. J Ayub Med Coll Abbottabad. 2019;31:336-339.

21. Afra TP, Razmi T, Narang M, Dogra T, Kumar A. Topical tazarotene gel, $0.1 \%$, as a novel treatment approach for atrophic postacne scars: a randomized active-controlled clinical trial. JAMA Facial Plast Surg. 2019;21:125-132. doi:10.1001/jamafacial.2018.1404

22. Minh PPT, Dang Bich D, Van Nguyen TH, et al. Microneedling therapy for atrophic acne scar: effectiveness and safety in Vietnamese patients. Open Access Maced J Med Sci. 2019;7 (2):293-297. doi:10.3889/oamjms.2019.098

23. Kim CNT, Thi LP, Nguyen Van T, et al. Successful treatment of facial atrophic acne scars by fractional radiofrequency microneedle in Vietnamese patients. Open Access Maced J Med Sci. 2019;7 (2):192-194. doi:10.3889/oamjms.2019.002

24. Saadawi AN, Esawy AM, Kandeel AH, El-Sayed W. Microneedling by dermapen and glycolic acid peel for the treatment of acne scars: comparative study. J Cosmet Dermatol. 2019;18(1):107-114. doi: $10.1111 /$ jocd. 12827

25. Bandral MR, Padgavankar PH, Japatti SR, et al. Clinical evaluation of microneedling therapy in the management of facial scar: a prospective randomized study. J Maxillofac Oral Surg. 2019;18 (4):572-578. doi:10.1007/s12663-018-1155-7

26. El-Domyati M, Moftah NH, Nasif GA, et al. Amniotic fluid-derived mesenchymal stem cell products combined with microneedling for acne scars: a split-face clinical, histological, and histometric study. $J$ Cosmet Dermatol. 2019. doi:10.1111/jocd.13039

27. Bulbul Baskan E, Akin Belli A. Evaluation of the efficacy of microneedle fractional radiofrequency in Turkish patients with atrophic facial acne scars. J Cosmet Dermatol. 2018. doi:10.1111/jocd.12812

28. Elawar A, Dahan S. Non-insulated fractional microneedle radiofrequency treatment with smooth motor insertion for reduction of depressed acne scars, pore size, and skin texture improvement: a preliminary study. J Clin Aesthet Dermatol. 2018;11:41-44.

29. Al Qarqaz F, Al-Yousef A. Skin microneedling for acne scars associated with pigmentation in patients with dark skin. J Cosmet Dermatol. 2018;17(3):390-395. doi:10.1111/jocd.12520

30. Kwon HH, Park HY, Choi SC, et al. Novel device-based acne treatments: comparison of a 1450-nm diode laser and microneedling radiofrequency on mild-to-moderate acne vulgaris and seborrhoea in Korean patients through a 20-week prospective, randomized, split-face study. J Eur Acad Dermatol Venereol. 2018;32:639-644.

31. El-Domyati M, Abdel-Wahab H, Hossam A. Microneedling combined with platelet-rich plasma or trichloroacetic acid peeling for management of acne scarring: a split-face clinical and histologic comparison. J Cosmet Dermatol. 2018;17:73-83. doi:10.1111/jocd. 12459 
32. Ibrahim MK, Ibrahim SM, Salem AM. Skin microneedling plus platelet-rich plasma versus skin microneedling alone in the treatment of atrophic post acne scars: a split face comparative study. J Dermatolog Treat. 2018;29(3):281-286. doi:10.1080/09546634.2017.1365111

33. Rana S, Mendiratta V, Chander R. Efficacy of microneedling with $70 \%$ glycolic acid peel vs microneedling alone in treatment of atrophic acne scars-a randomized controlled trial. $J$ Cosmet Dermatol. 2017;16(4):454-459. doi:10.1111/jocd.12377

34. Kwon HH, Park H, Choi S, et al. Combined fractional treatment of acne scars involving non-ablative 1550 -nm erbium-glass laser and micro-needling radiofrequency: a $16-$ week prospective, randomized split-face study. Acta Derm Venereol. 2017;97(8):947-951. doi:10.2340/00015555-2701

35. Faghihi G, Poostiyan N, Asilian A, et al. Efficacy of fractionated microneedle radiofrequency with and without adding subcision for the treatment of atrophic facial acne scars: a randomized split-face clinical study. J Cosmet Dermatol. 2017;16(2):223-229. doi:10.1111/ jocd.12346

36. Pudukadan D. Treatment of acne scars on darker skin types using a noninsulated smooth motion, electronically controlled radiofrequency microneedles treatment system. Dermatol Surg. 2017;43 (Suppl 1):S64-S69. doi:10.1097/DSS.0000000000000894

37. Kroepfl L, Emer JJ. Combination therapy for acne scarring: personal experience and clinical suggestions. $J$ Drugs Dermatol. 2016;15:1413-1419.

38. Yadav S, Dogra S, Cutaneous A. Reaction to microneedling for postacne scarring caused by nickel hypersensitivity. Aesthet Surg J. 2016;36:NP168-NP170. doi:10.1093/asj/sjv229

39. Cachafeiro T, Escobar G, Maldonado G, Cestari T, Corleta O. Comparison of nonablative fractional erbium laser $1340 \mathrm{~nm}$ and microneedling for the treatment of atrophic acne scars: a randomized clinical trial. Dermatol Surg. 2016;42(2):232-241. doi:10.1097/DSS.0000000000000597

40. Asif M, Kanodia S, Singh K. Combined autologous platelet-rich plasma with microneedling verses microneedling with distilled water in the treatment of atrophic acne scars: a concurrent split-face study. J Cosmet Dermatol. 2020;145(4):434-443. doi:10.1111/jocd.12207

41. Park JY, Lee EG, Yoon MS, Lee HJ. The efficacy and safety of combined microneedle fractional radiofrequency and sublative fractional radiofrequency for acne scars in Asian skin. $J$ Cosmet Dermatol. 2019;21(2):102-107. doi:10.1111/jocd.12195

42. Naouri M, Mazer JM. Non-insulated microneedle fractional radiofrequency for the treatment of scars and photoaging. J Eur Acad Dermatol Venereol. 2016;30(3):499-502. doi:10.1111/jdv.12890

43. Weiss RA, Weiss MA, Halvorson CR, Flor Mayoral MD, Ross EV. Treatment of acne scars with high intensity focused radio frequency. J Drugs Dermatol. 2015;14:1065-1068.

44. Dogra S, Yadav S, Sarangal R. Microneedling for acne scars in Asian skin type: an effective low cost treatment modality. $J$ Cosmet Dermatol. 2014;13(3):180-187. doi:10.1111/jocd.12095

45. Chandrashekar BS, Sriram R, Mysore R, Bhaskar S, Shetty A. Evaluation of microneedling fractional radiofrequency device for treatment of acne scars. J Cutan Aesthet Surg. 2014;7(2):93-97. doi:10.4103/0974-2077.138328

46. Garg S, Baveja S. Combination therapy in the management of atrophic acne scars. J Cutan Aesthet Surg. 2014;7(1):18-23. doi:10.4103/0974-2077.129964

47. Vejjabhinanta V, Wanitphakdeedecha R, Limtanyakul P, Manuskiatti W. The efficacy in treatment of facial atrophic acne scars in Asians with a fractional radiofrequency microneedle system. J Eur Acad Dermatol Venereol. 2014;28(9):1219-1225. doi: $10.1111 /$ jdv. 12267

48. Fabbrocini G, De Vita V, Monfrecola A, et al. Percutaneous collagen induction: an effective and safe treatment for post-acne scarring in different skin phototypes. J Dermatolog Treat. 2014;25(2):147-152. doi:10.3109/09546634.2012.742949
49. Pahwa M, Pahwa P, Zaheer A. "Tram track effect" after treatment of acne scars using a microneedling device. Dermatol Surg. 2012;38 (7pt1):1107-1108. doi:10.1111/j.1524-4725.2012.02441.x

50. Cho SI, Chung BY, Choi MG, et al. Evaluation of the clinical efficacy of fractional radiofrequency microneedle treatment in acne scars and large facial pores. Dermatol Surg. 2016;42(7pt1):1017-1024. doi:10. $1111 / \mathrm{j} .1524-4725.2012 .02402 . x$

51. Sharad J. Combination of microneedling and glycolic acid peels for the treatment of acne scars in dark skin. J Cosmet Dermatol. 2011;10:317-323.

52. Casabona GR, Giacomo TB. Improving the appearance of surgical facial scars with incobotulinumtoxinA and microneedling. J Drugs Dermatol. 2020;19(6):611-615. doi:10.36849/JDD.2020.4772

53. Agamia NF, Sorror O, Alrashidy M, Tawfik AA, Badawi A. Clinical and histopathological comparison of microneedling combined with platelets rich plasma versus fractional erbium-doped yttrium aluminum garnet (Er: YAG) laser $2940 \mathrm{~nm}$ in treatment of atrophic post traumatic scar: a randomized controlled study. J Dermatolog Treat. 2020;1-8. doi:10.1080/09546634.2020.1729334

54. Abdel-Magiud EM, Taha EA, Bakr RM, et al. Effects of different therapeutic modalities for postacne scars on circulating collagen III. J Cosmet Dermatol. 2020;19(6):1517-1521. doi:10.1111/jocd.13184

55. Ali B, ElMahdy N, Elfar NN. Microneedling (Dermapen) and Jessner's solution peeling in treatment of atrophic acne scars: a comparative randomized clinical study. J Cosmet Laser Ther. 2019;21(6):357-363. doi:10.1080/14764172.2019.1661490

56. Tan C, Yeo Chen Long D, Cao T, et al. Drug-free microneedles in the treatment of keloids: a single-blinded intraindividual controlled clinical trial. Br J Dermatol. 2018;179(6):1418-1419. doi:10.1111/ bjd. 17078

57. Busch K-H, Aliu A, Walezko N, Aust M. Medical needling: effect on moisture and transepidermal water loss of mature hypertrophic burn scars. Cureus. 2018;10:e2365.

58. Yeo DC, Balmayor ER, Schantz J-T, Xu C. Microneedle physical contact as a therapeutic for abnormal scars. Eur $J$ Med Res. 2017;22:28. doi:10.1186/s40001-017-0269-6

59. Yoo KH, Ahn GR, Seok J, Jang Y-J, Kim BJ. Successful treatment of smallpox scars by using radiofrequency device with single microneedle. Int Wound J. 2018;179(6):1399-1400. doi:10.1111/ iwj. 12771

60. Mahadevappa OH, Mysore V, Viswanath V, et al. Surgical outcome in patients taking concomitant or recent intake of oral isotretinoin: a multicentric study-ISO-AIMS study. J Cutan Aesthet Surg. 2016;9 (2):106-114. doi:10.4103/0974-2077.184054

61. Chae WS, Seong JY, Jung HN, et al. Comparative study on efficacy and safety of $1550 \mathrm{~nm}$ Er:Glass fractional laser and fractional radiofrequency microneedle device for facial atrophic acne scar. $J$ Cosmet Dermatol. 2015;14(2):100-106. doi:10.1111/jocd.12139

62. Costa IMC, Costa MC. Microneedling for varicella scars in a dark-skinned teenager. Dermatol Surg. 2014;40(3):333-334. doi: $10.1111 /$ dsu. 12396

63. Šuca H, Zajíček R, Vodsloň Z. Microneedling - a form of collagen induction therapy - our first experiences. Acta Chir Plast. 2017;59:33-36.

64. FDA. Regulatory considerations for microneedling devices. 2018. Available from: https://www.fda.gov/regulatory-information/searchfda-guidance-documents/regulatory-considerations-microneedlingdevices. Accessed December 4, 2020.

65. Zeitter S, Sikora Z, Jahn S, et al. Microneedling: matching the results of medical needling and repetitive treatments to maximize potential for skin regeneration. Burns. 2014;40(5):966-973. doi:10.1016/j. burns.2013.12.008

66. El-Domyati M, Barakat M, Awad S, et al. Multiple microneedling sessions for minimally invasive facial rejuvenation: an objective assessment. Int J Dermatol. 2015;54(12):1361-1369. doi:10.1111/ ijd.12761 
67. Gualeni B, Coulman SA, Shah D, et al. Minimally invasive and targeted therapeutic cell delivery to the skin using microneedle devices. Br J Dermatol. 2018;178(3):731-739. doi:10.1111/bjd 15923

68. Xie Y, Wang H, Mao J, et al. Enhanced in vitro efficacy for inhibiting hypertrophic scar by bleomycin-loaded dissolving hyaluronic acid microneedles. J Mater Chem B. 2019;7(42):6604-6611. doi:10.10 39/C9TB01449G
69. Lin S, Quan G, Hou A, et al. Strategy for hypertrophic scar therapy: improved delivery of triamcinolone acetonide using mechanically robust tip-concentrated dissolving microneedle array. $J$ Control Release. 2015;14:69-82. doi:10.1016/j.jconrel.2019.05.038

70. Weiner SF. Radiofrequency microneedling: overview of technology, advantages, differences in devices, studies, and indications. Facial Plast Surg Clin North Am. 2019;27:291-303. doi:10.1016/j.fsc.2019.03.002

\section{Publish your work in this journal}

Clinical, Cosmetic and Investigational Dermatology is an international, peer-reviewed, open access, online journal that focuses on the latest clinical and experimental research in all aspects of skin disease and cosmetic interventions. This journal is indexed on CAS.
The manuscript management system is completely online and includes a very quick and fair peer-review system, which is all easy to use. Visit http://www.dovepress.com/testimonials.php to read real quotes from published authors.

Submit your manuscript here: https://www.dovepress.com/clinical-cosmetic-and-investigational-dermatology-journal 